\title{
ESTUDO ECOLÓGICO DA TAXA DE MORTALIDADE CAUSADA POR DOENÇA DE CHAGAS NO NORDESTE BRASILEIRO
}

\section{Pietra Zava Lorencinii ${ }^{1}$, Sara da Silva Pereira ${ }^{2}$; Geisa Santana de Oliveira ${ }^{3}$; Lucas Dalvi Armond Rezende ${ }^{4}$; Isadora Bianchi Daré5; Maria Eduarda Morais Hibner Amaral ${ }^{6}$; Paula de Souza Silva Freitas ${ }^{7}$; Daniel Altoé Sossai ${ }^{8}$; Anna Carolina Dockhorn de Menezes Carvalho Costa ${ }^{9}$}

1,2,3,4,5,6,7Universidade Federal do Espírito Santo (UFES), Vitória, ES.

${ }^{7}$ Universidade Federal do Espírito Santo (UFES), Vitória, ES.

${ }^{8,9}$ Escola Superior de Ciências da Santa Casa de Misericórdia de Vitória (EMESCAM), Vitória, ES.

DOI: 10.47094/IICNNESP.2021/80

PALAVRAS-CHAVE: Doença de Chagas. Mortalidade. Nordeste.

ÁREA TEMÁTICA: Epidemiologia.

\section{INTRODUÇÃO}

O Brasil apresenta uma constante taxa de mortalidade causada por doenças infecciosas e parasitárias, segundo Souza HP, et. al. (2020). A região nordeste, por sua vez, destaca-se pelos elevados índices de óbitos em decorrência da Doença de Chagas (CORREIA, et al. 2021). Essa doença é causada pelo protozoário flagelado Trypanosoma cruzi, que pode ser encontrado nas fezes de insetos da subfamília Triatominae, popularmente conhecido como Barbeiro. Sua transmissão ocorre por contato direto com o protozoário, por meio de transfusão sanguínea, consumo de alimentos ou água contaminados pelas fezes do animal, por transmissão vertical e até mesmo em casos de transplante de órgãos. Existem casos assintomáticos durante a fase aguda, o que dificulta o diagnóstico e pode levar a pessoa a evoluir para a fase crônica, em que há a ocorrência de problemas cardíacos e/ou digestivos que podem levar à morte em casos mais graves (ORTIZ, et al. 2019). Por isso, objetiva-se analisar o perfil epidemiológico dos óbitos ocasionados pela Doença de Chagas na região Nordeste no período de 2010 a 2019.

\section{METODOLOGIA}

Realizou-se um estudo ecológico a partir dos dados do DATASUS, provenientes do sistema de informação de mortalidade - SIM, em relação a população nordestina com faixa etária entre 40 e 80 anos ou mais. Os dados coletados foram referentes à taxa de mortalidade causada por doença infecciosa e parasitária do capítulo I da $10^{\mathrm{a}}$ revisão da classificação internacional de doenças, especificamente em relação a Doença de Chagas, no período de 2010 a 2019. 


\section{RESULTADOS E DISCUSSÕES}

Os determinantes sociais de saúde (DSS), utilizados para relacionar as condições de saúde da população de acordo com o contexto de vida individual, são fundamentais para a garantia de uma saúde pública que atenda às necessidades de cada paciente, uma vez que é impossível desvincular a ciência médica da social (BUSS; PELLEGRINI FILHO, 2007). Desse modo, a correlação entre a taxa de mortalidade causada por doenças infecciosas e parasitárias, como a Doença de Chagas, e os determinantes sociais de saúde é imprescindível para compreensão da realidade no nordeste brasileiro.

A Doença de Chagas, por sua vez, apesar de englobar estratégias de saúde a fim de evitar a sua incidência, ainda apresenta números elevados de mortalidade na região Nordeste como reflexo das condições sociais, culturais, econômicas e étnicas/raciais. Em virtude desse cenário, entre os anos de 2010 e 2019, registrou-se um total de 9.773 óbitos ocasionados por esse protozoário, com notória constância dos números ao longo dos anos. Em relação ao local de ocorrência dos óbitos, nota-se que, desse total, 2.803 ocorreram no próprio domicílio, o que sugere um distanciamento do indivíduo em relação ao sistema de saúde. No que tange a cor, 1.779 óbitos envolviam pessoas pretas, o que reflete a desigualdade social e histórica ainda presente na região. Ademais, 3.194 dos casos de mortes notificados eram de indivíduos sem nenhuma escolaridade, o que preconiza a falta de conhecimento em relação à doença.

Portanto, o perfil epidemiológico da taxa de mortalidade causada pela Doença de Chagas na região Nordeste enquadra-se, significativamente, com os parâmetros dos determinantes sociais de saúde, uma vez que, apesar da idade, sexo, fatores hereditários e estilo de vida influenciarem, outros fatores exercem grande efeito nos indivíduos. A rede comunitária e social, por exemplo, é importante para a difusão de experiências e para fornecer ajuda. As condições de vida e ambientais, como habitação, água e esgoto, são necessárias para interromper o ciclo das doenças parasitárias e infecciosas. E, por fim, não se pode negar o efeito das condições educacionais, socioeconômicas e culturais nos registros de mortalidade no Brasil.

\section{CONCLUSÃO}

Diante da taxa de mortalidade ocasionada pela Doença de Chagas na região Nordeste, é importante refletir e executar intervenções com intuito de minimizar os efeitos dos determinantes sociais. Dentre as medidas mais importantes estão o controle do vetor, que diminui as chances desses insetos transmitirem a doença para a população, além de ações educativas para ensinar os indivíduos sobre a prevenção da doença. A educação é um componente da promoção da saúde e não deve ser resumida apenas como informação, que se transmite de maneira unidirecional com palestrantes e panfletos, ou seja, ela demanda trocas culturais entre profissionais da saúde, trabalhadores rurais, famílias e comunidades. O desenvolvimento desse processo com êxito contribuirá para diminuição da transmissão e, consequentemente, de mortes advindas da Doença de Chagas. 


\section{PRINCIPAIS REFERÊNCIAS}

BUSS, Paulo Marchiori; PELLEGRINI FILHO, Alberto. A Saúde e seus Determinantes Sociais. Physis: Rev. Saúde Coletiva, Rio de Janeiro, v. 1, n. 17, p. 77-93, 15 mar. 2007.

CORREIA, Jennifer Rodrigues; RIBEIRO, Suzana Cristina Silva; ARAðJO, Lorena Vieira Fernandez de; SANTOS, Mariane Costa; ROCHA, Thiago Reis; VIANA, Emanuelle Almeida Silva; CAIRES, Poliana Terra Pires Ribeiro Coelho; CORRêA, Shesllen Mikaelly Cruz; PINHEIRO, Taise Gonçalves; CARVALHO, Lenise Costa de. Doença de Chagas: aspectos clínicos, epidemiológicos e fisiopatológicos. Revista Eletrônica Acervo Saúde, [S.L.], v. 13, n. 3, p. 6502, 2 mar. 2021. Revista Eletrônica Acervo Saúde. http://dx.doi.org/10.25248/reas.e6502.2021.

DEPARTAMENTO DE INFORMÁTICA DO SUS - DATASUS. Informações de Saúde, Estatísticas Vitais: banco de dados. Disponível em: < http://www2.datasus.gov.br/DATASUS/index.php? area $=0205>$ Acesso em: 19 abril. 2021.

GRYNSZPAN, Danielle. Portal Doença de Chagas: educação e promoção da saúde. Educação e Promoção da Saúde. 2017. Disponível em: <http://chagas.fiocruz.br/> Acesso em: 28 abr. 2021.

ORTIZ, Jessica Vanina et al. Avaliação Cardíaca na Fase Aguda da Doença de Chagas com Evolução Pós-Tratamento em Pacientes Atendidos no Estado do Amazonas, Brasil. Arq. Bras. Cardiol., São Paulo , v. 112, n. 3, p. 240-246, Mar. 2019. Disponível em: <http://www.scielo.br/scielo. php?script=sci_arttext\&pid=S0066-782X2019000300240\&lng=en\&nrm=iso $>$. Acesso em: 29 abr. 2021.

Souza HP, Oliveira WTGH, Santos JPC, Toledo JP, Ferreira IPS, Esashika SNGS, et al. Doenças infecciosas e parasitárias no Brasil de 2010 a 2017: Aspectos para vigilância em saúde. Rev Panam Salud Publica. 2020;44:e10. https://doi.org/10.26633/RPSP.2020.10. 